\title{
Influence of Gas Pressure and RF Power on the Structural and Mechanical Properties of Germanium thin Films
}

Nurul Assikin Ariffin

Universiti Putra Malaysia

Siti Nor Aliffah Mustaffa

Universiti Putra Malaysia

Nizam Tamchek

Universiti Putra Malaysia

Suresh Sagadevan

University of Malaya

Suriati Paiman ( $\nabla$ suriati@upm.edu.my )

Universiti Putra Malaysia https://orcid.org/0000-0002-1459-3092

\section{Research Article}

Keywords: Radiofrequency magnetron sputtering, gas pressure, radio frequency power, thickness and surface roughness

Posted Date: March 15th, 2021

DOl: https://doi.org/10.21203/rs.3.rs-285400/v1

License: (c) (i) This work is licensed under a Creative Commons Attribution 4.0 International License. Read Full License 


\section{Abstract}

Germanium thin film was deposited on a glass substrate by radio frequency magnetron sputtering technique toward nanowires growth for solar cell application. Germanium thin films were deposited at room temperature. The gas pressures and radiofrequency power were varied from 50 to $100 \mathrm{Watt}$ and 5 to $15 \mathrm{mTorr}$, respectively, with constant deposition time. The thickness of the deposited thin film was investigated using a high surface profilometer. Meanwhile, its structural properties were characterized using atomic force microscopy technique. Results showed that the surface roughness of the Germanium thin film decreased with the increase of gas pressure and radio frequency power. All the thin films formed were amorphous which were confirmed with X-ray diffraction analysis. The experiment results indicated that thin films deposit at $15 \mathrm{mTorr}$ produced $114.76 \mathrm{~nm}$ of thickness and $61.9 \mathrm{~nm}$ of surface roughness. Meanwhile, Ge thin film deposited at 100-Watt radio frequency power had $10.2 \mathrm{~nm}$ of surface roughness. Therefore, growth parameters must be optimized in order to obtain the desired surface roughness which then can be used for the growth of nanowires for solar cell application.

\section{Introduction}

Experimentally, numerous studies have turned their focus on how to achieve a high-quality Germanium thin film through various PVD techniques, for instance by reactive sputtering [1], hollow cathode reactive sputtering [2], co-sputtering [3], and RF magnetron sputtering [4]. However, the deposition of a material using a sputtering process is limited by low deposition rates, low ionization probability, and substrate heating effects [1]. These limitations have been overcome by the development of the magnetron sputtering technique, where a permanent magnet was used to collimate the electron beam hitting the target material, thereby increasing the number of electrons that takes part in the ionization process [5]. Hence, the magnetron sputtering increases the probability of electrons striking the argon atoms and increase the sputtering efficiency [6]. Most of these studies demonstrate how deposition parameters can be tailored with the aim of obtaining high-quality Ge thin films. One important factor for the successful use of Ge thin films in the device is the precise control of thin-film deposition to achieve the desired surface roughness of the thin film. Several studies have reported the effect of root-mean-square (RMS) surface roughness on the quality of the thin film $[7,8]$. In particular, controlling the surface roughness of the thin films is a primary motivation in Ge thin film research [9]. Researchers are still investigating the sputtering deposition to understand and explain the experimental results of thin films under several deposition parameters such as gas pressure and RF power. Hence, in this paper, we demonstrate Ge thin film deposited on a glass substrate at room temperature by varying its RF power and gas pressure to optimize its surface roughness and thickness. This result may have significance to the growth of nanowires toward solar cell applications.

\section{Experimental Procedure}

Ge thin film was deposited on glass substrates by RF magnetron sputtering system. The target used was metallic Ge disk with 99.999 \% of purity, in argon ambient at room temperature for an hour. The chamber 
was pumped down to a base pressure less than $1 \times 10^{-3} \mathrm{mTorr}$ before deposition. The argon gas flow was controlled using mass flow controllers and the gas pressure was varied as 5,10 , and 15 mTorr, with a plasma excitation frequency of $13.56 \mathrm{MHz}$. The RF power also was varying at different power of 50,75 , and 100 Watt. The glass substrate was cleaned with de-ionized water and acetone, and then it was heated for a few minutes to remove any residual moisture before deposition. The morphology of the thin film was analyzed by atomic force microscopy (AFM). The AFM measurement was performed using noncontact mode and the scan area was $10 \times 10 \mu \mathrm{m}^{2}$ to determine the RMS surface roughness value of the thin film. Meanwhile, the thin film thickness was measured using a high surface profilometer (Ambios, XP-200). The structural and crystalline properties of the layer were characterized using X-ray Diffraction (Philips, PW3040/60).

\section{Results And Discussion}

\subsection{Thickness measurements}

The gas pressure and radio frequency (RF) power have a very significant effect on the Ge thickness and deposition rate. Table.1 shows the thickness and deposition rate of Ge thin film investigated in this study. The results were taken using a stylus high surface profilometer. As the results showed, the Ge thin film thickness and the deposition rate value decreased with the increase of gas pressure.

Table.1. Thicknesses of Ge thin film sputter with different gas pressure

\begin{tabular}{|llll|}
\hline Pressure (mTorr) & RF power (Watt) & Thickness $(\mathrm{nm})$ & Deposition rate $(\mathrm{nm} / \mathrm{min})$ \\
\hline 5 & 25 & $235.37 \pm 4.11$ & 3.922 \\
\hline 10 & & $182.34 \pm 3.37$ & 3.039 \\
\hline 15 & & $114.76 \pm 2.89$ & 1.913 \\
\hline & 50 & $114.16 \pm 2.39$ & 1.912 \\
\hline & 75 & $154.92 \pm 2.94$ & 2.582 \\
\hline
\end{tabular}

The atoms can be ejected or sputtered from solids by bombarding their surface with energetic ions during the deposition. Throughout the process, a working gas (typically argon) was introduced and it served as a medium in which an electrical discharge was initiated and sustained. After the visible glow, discharge or plasma is maintained between the electrodes, it is observed that current flowed through the substrate. The positive gas ions in the discharge struck the cathode and physically ejected or sputtered target atoms through the momentum transfer method. These atoms entered and passed through the discharge region to eventually get deposited on the substrate. 
Based on the results investigated, the lower gas pressure of 5 mTorr would have a higher thickness of $235.37 \mathrm{~nm}$ (Figure.1). However, the reduction in gas pressure increased the energetic particle bombardment which in turn densified the thin film [10]. The densification of thin-film led to the reduction of a thin-film thickness [10]. Thus, high ion energies could be maintained at low pressure in order to prevent too many atom-gas collisions after ejection from the target. Moreover, a lower gas pressure, the number of sputter atoms decreased due to less collisions between gas and atom in the chamber [11]. The collision probability is small at a lower pressure due to the larger mean-free path with causes loss of electrons to the wall chamber [10]. Therefore, overall ionization efficiency became low. Nevertheless, the contradiction of the results was obtained possibly due to the interaction by a collision between the target atoms and ions produced neutral atoms during the sputter deposition process. These neutral atoms would collide elastically with another atom in the chamber and prevent atoms to be sputtered at the surface of the glass substrate. Besides that, the sputtered atom may get reflected from the target and the wall chamber continuously.

Additionally, at lower pressure, the number of collisions between the target atoms and argon ions is lower since it has higher mean-free path. This will cause the deposition rate to increase. Diffusion length of adsorbed species can compensate, improving step coverage and uniformity on a surface of glass substrate which also supported by Gatzen at al. [13].

At a higher gas pressure of $15 \mathrm{mTorr}$ (Figure 1), Ge thin film thickness was found to decrease at 114.76 $\mathrm{nm}$. This could be at higher gas pressure and attribute to many scattering collisions that occur so that the energetic-atom bombardment of the growing thin film is slowing down. Since a lot of scattering occurred, a lot of atoms struck the surface of the glass substrate and would experience a bigger mean-free path as the gas pressure was increased. The collision probability was small due to the large mean-free path which caused the loss of electrons to the chamber walls [12]. Electrons having the bigger mean-free path can store higher energy before the collision with other molecules, and they can produce active ionization by collision. Moreover, at higher gas pressure, the growth rate decreased due to the improvement of sputtered particles in the chamber [11].

It can be seen that thickness uniformity is mainly influenced by the deposition rate [13]. The deposition rate is defined as the thickness deposited atom (in $\mathrm{nm}$ ) per unit deposition time (in minutes). Consequently, lower gas pressure will cause a high deposition rate. As mention earlier, the collision increases due to many neutral atoms striking the atoms and ions in the chamber causing the mean-free path of the sputtered atoms decreasing. The atoms sputtered on the glass substrate are compressed further and having a lower thickness with more uniformity.

On the other hand, the low deposition rate that occurs at higher gas pressure may cause collisions between ions to decrease and atoms possibly may get deflected straight back onto the target. Therefore, the number of ions being sputtered is lower on the glass substrate in the chamber. This consequently causes the Ge thin film thickness to decrease as the gas pressure increases, which is similar to the reports of the previous works cited in the literature [14-16]. 
The RF power is involved in alternating the electrical potential of the current in the vacuum environment at radio frequencies of $13.56 \mathrm{MHz}$ to avoid charge building up on certain types of sputtering target materials. The use of RF power in the magnetrons sputtering process eventually causes the electrons to become exceedingly energized in the plasma, creating a "hammer effect" in which the ions bring themselves into the substrate with greater force. Thus, this enables them to pack down tight to create very flat and uniform thin films with low surface roughness. The RF power has a very significant effect on the Ge thickness and deposition rate in this study. Figure. 2 shows the graph of thickness and deposition rate for Ge thin film at different RF power. Based on the results shown, as we can see the Ge thin film thickness and deposition rate increased with the increase of RF power.

The deposition at low RF power of 25 Watt produces a thickness of $114.76 \mathrm{~nm}$ thin film, with 1.913 $\mathrm{nm} / \mathrm{min}$ of deposition rate. It is possible that the low deposition thickness caused by atoms bombards the substrate in the chamber at low energy, which caused relatively random and slower ions bombardment to the surface of the glass substrate and caused the Ge thin film thickness to become lower. The mean-free path of the ions also increases, which leads the kinetic energy to be reduced respectively at a lower RF power.

Meanwhile, high RF power of 100 Watt leads to a high thickness of $232.32 \mathrm{~nm}$ and a high deposition rate of $3.872 \mathrm{~nm} / \mathrm{min}$. Belfedal et al. reported that a high deposition rate with of the material is due to the plasma discharge at high RF power in the sputtering system. Within that system, the energy is more efficiently coupled to the plasma through the bulk electrons that gain energy from the electrical field generated between the electrodes [14]. The energy gain at high RF power increases the kinetic energy of the ions allowing ions to bombard more on the glass substrate and increasing the thin film thickness. Besides, that the energy of electrons and ions in glow discharge plasma will increase with the increment in the sputtering power [12]. This is possibly due to the energy of the sputtered atom which is obtained from the positive ions colliding with the target, increasing the sputtering power. Therefore, the energy of the sputtered atom arriving at the substrate increases with the increases in power. Moreover, it is also possible that the highly energized electron with increasing sputtering power will bombard the surface of the growing thin film of the substrate, providing in the form of thermal energy. This energy might act as additional energy to promote the growth of the thin film thickness [12]. Therefore, it can be concluded that as the sputtering power increases, the number and momentum of the sputtering particles will also increase.

\subsection{Surface roughness}

Table. 2 shows the data analysis of surface roughness obtained from AFM characterization at different gas pressures and RF powers. As we can see, the RMS surface roughness and maximum peak value are increased with the increasing gas pressure and RF power.

Table.2. Analysis of AFM characterization of Ge thin films at different gas pressures 


\begin{tabular}{|llll|}
\hline Pressure (mTorr) & RF power (Watt) & RMS surface roughness $(\mathrm{nm})$ & Maximum peak value $(\mathrm{nm})$ \\
\hline 5 & 25 & $1.898 \pm 0.073$ & 67.6 \\
\hline 10 & & $12.141 \pm 0.524$ & 84.5 \\
\hline 15 & & $61.910 \pm 3.865$ & 397.0 \\
\hline 15 & 50 & $2.630 \pm 0.021$ & 98.0 \\
\hline & 75 & $4.109 \pm 0.870$ & 86.0 \\
\hline
\end{tabular}

At low gas pressure, the bombarding atoms and ions have high kinetic energy and hence more atoms being sputtered on the glass substrate. These cause the deposited thin film to become denser and more fibrous in structure, consequently causing the deposited surface thin film to become smoother. However, when the gas pressure increases, the energy of the bombarding atoms is reduced due to the collision between atoms and neutrals in the chamber. Therefore, this causes the thin film to become more porous and hence tensile [17]. AFM images show in Figures.3 (a), (b), and (c) show the $10 \times 10 \mu \mathrm{m}^{2}$ of Ge thin film deposited on the glass substrate at different gas pressures. AFM analysis shows that the increase of maximum peak value causes more protruding peak formed on the surface of the thin film. The growth of bubbles and sharp pits clearly can be observed on all the surfaces of Ge thin films deposited on the glass substrate.

From the figure shown, the surface condition seems smooth at a low gas pressure. It can be clearly seen that the surface has small protruding peaks. However, at higher gas pressure, the bubble and protruding peak formation become bigger and it is believed that the thin film is not densified or diffused into the substrate. Moreover, higher gas pressure possibly can cause surface damage to the deposited Ge thin film since the kinetic energy is higher as the gas pressure increases $[11,16]$. Besides, the increase of RMS surface roughness indicates that the surface becomes rough and coarse. Coarsening in thin film refers to the competitive growth process being some islands or bubble growth at the expense of another, to minimize the total surface energy. This leads to increases in the maximum value and results in decreasing the mean area density of the thin film [8]. The coarsening is possibly due to the combination of different mechanisms, such as adatom diffusion or inter-diffusion.

The images having some shadowing effect during the AFM analysis, where the dragging motion clearly visible in the image may cause by the probe tip, combining with adhesive forces between the tip and the surface. This can cause substantial damage to both the thin film surface and the probe and create artificial data, such as shadowing effect. This also can be caused by the used of strong repulsive forces acting between the tip and the thin film surface.

Since at low RF power of 50 Watt there occurs low atoms bombardment in the chamber, the atoms move slower due to lower kinetic energy causing low atoms bombardment on the surface of the glass 
substrate. Electron having longer mean-free path can store higher energy before collisions with argon atom and they can produce active ionization by the collision. It follow many positive ions with higher energy can collide to target and sputter more Ge molecules from the target, giving an increment in deposition rate and improvement in thin film quality [15]. Moreover, this leads to the lower RMS surface roughness and the maximum peak value of $2.63 \mathrm{~nm}$ and $98.0 \mathrm{~nm}$ respectively. However, the kinetic energy of the atoms increases as the RF power increases, thereby causing the bombardment of atom on the surface of the thin film to increase. Atoms bombardment with high kinetic energy causes a large force to strike the surface possibly causing damage and increasing the value of RMS surface roughness of the thin film. Therefore, as can be seen, the RMS surface roughness is $10.283 \mathrm{~nm}$ with the maximum peak value of $188.0 \mathrm{~nm}$ at high RF power of 100 Watt.

Generally, the maximum peak value increased with the increase of RF power at constant gas pressure. As discussed before, this was caused by many ions collisions reaching the surface of the glass substrate when higher RF power was applied. The RF power also causes the electrons to become energized in the plasma, which leads ions to bring themselves down onto the glass substrate with a great force. This allows ions to create a high peak on the glass substrate. The particle energy increases during the deposition process at the higher RF power. Hence, it may cause the average peak value and maximum peak value to increase.

Figures.4 (a), (b), and (c) show the $10 \times 10 \mu \mathrm{m}^{2}$ AFM scan images of the as-deposited Ge thin film deposited at different RF powers. Figure 4 (a) shows the formation of high sharp peaks with a maximum peak was observed at $98 \mathrm{~nm}$. Even though it has a few peaks, the surface seems smoother without any formation of bubbles, clusters or an island. This is caused by low atoms bombardment during the deposition process. The formation of peak disappears, and the surface becomes more consistent, as the RF power is increased. Thus, the RMS surface roughness may increase due to the increased thickness and surface damages as mentioned in the previous section. Lin et al., make a supporting statement that the increase of the RF power will provide more energy to surface atoms and further contribute to the possibility of producing a surface with a higher RMS surface roughness, thickness, and maximum peak value [16].

\subsection{Structural properties}

Phase analysis in this study was characterized using XRD. Figures.5 (a) and (b), show the XRD analysis of the as-deposited Ge thin film at different gas pressures and RF powers. Based on the figure shown, all as-deposited Ge thin films obtained from this study are amorphous thin films. As we can see, all deposited Ge thin films exhibit amorphous behaviour with no characteristic peaks being detected on the graph. A hump around 20 to $25^{\circ}$ as shown in Figures. 5 (a) and (b), indicates the glass substrate used in the study [3]. All the thin film samples were deposited at room temperature. Particularly, amorphous $\mathrm{Ge}$ thin film is in metastable condition even at room temperature and it has poor stability [15]. Metastable condition refers to the tendency of atoms to stable states that have the lowest free energy and are defined by the equilibrium phase diagram. 
A crystal is composed of atoms periodically arranged in 3D space. However, amorphous materials do not possess the periodicity and atoms are randomly distributed in 3D space. The scattering of X-rays by atoms is the point to be considered in this case. When there is a periodic arrangement of atoms, the $\mathrm{x}$ rays will be scattered only in certain directions when they hit the formed lattice planes. This will cause high-intensity peaks with the width of the peaks depending on other variables. As for the amorphous phase, the X-rays will be scattered in many directions leading to a large bump distribution in a wide range of angles $(2 \theta)$ instead of high intensity with narrower peaks in the crystal structure. Therefore, the diffraction of Ge thin film in this study does not show sharp Bragg peaks in the XRD analysis. Also, the amorphous condition is said to be metastable solid with no long-range atomic order. Therefore, they produce only a broad scattering peak. This condition always occurs in the thin film material using glass as a substrate. The structure lacks symmetry and produces a broad diffraction peak compared to crystalline phase material. The broad peak may also affect the optical properties of the thin film.

\section{Conclusions}

In summary, Germanium thin film was deposited on a glass substrate by radio frequency magnetron sputtering technique. The gas pressure and radio frequency (RF) power had a very significant effect on the Ge thickness and deposition rate. As the results showed, the Ge thin film thickness and deposition rate value decreased with the increase of gas pressure. AFM images show Ge thin film being deposited on the glass substrate at different RF power and gas pressure. AFM analysis showed that the increase of maximum peak value caused more protruding peak formed on the surface of the thin film. Moreover, this led to the lower RMS surface roughness and the maximum peak value of $2.63 \mathrm{~nm}$ and $98.0 \mathrm{~nm}$ respectively. It was revealed that all thin films were amorphous phase by $\mathrm{X}$-ray diffraction analysis. The lowest RMS surface roughness obtained is $1.898 \mathrm{~nm}$ with a thickness of $235.27 \mathrm{~nm}$. It was found that the RMS surface roughness increased with the increase of gas pressure and RF power.

\section{Declarations}

\section{Competing interests:}

The authors declare no conflict of interest

\section{Acknowledgments}

The authors gratefully acknowledge the support from Universiti Putra Malaysia research grants 9457700 and 9456800 . We would also like to extend our appreciation to the Ministry of Education Malaysia (under the Fundamental Research Grant Scheme (FRGS) 5524429, 5540050 and the Exploratory Research Grant Scheme (ERGS) 5527188) for providing the financial support.

\section{References}


1. Che, Xing Sen, Zheng Tang Liu, Yang Ping Li, Ning Wang, and Zuo Xu. Effects of Methane Flow Rate on the Optical Properties and Chemical Bonding of Germanium Carbon Films Deposited by Reactive Sputtering. Vaccum, 90, 75-79(2013)

2. Soukup, R. J., N. J. lanno, G. Pribil, and Z. Hubicka. Deposition of High-Quality Amorphous Silicon, Germanium and Silicon-Germanium Thin Films by a Hollow Cathode Reactive Sputtering System. Surface and Coatings Technology 177-178: 676-81 (2004).

3. Tsao, Chao-yang, Jialiang Huang, Xiaojing Hao, Patrick Campbell, and Martin A Green. Solar Energy Materials \& Solar Cells Formation of Heavily Boron-Doped Hydrogenated Polycrystalline Germanium Thin Films by Co-Sputtering for Developing P + Emitters of Bottom Cells. Solar Energy Materials and Solar Cells, 95 (3), 981-85 (2011).

4. Liu, Ziheng, Xiaojing Hao, Anita Ho-baillie, Chao-yang Tsao, and Martin A Green. Cyclic Thermal Annealing on Ge / Si ( 100 ) Epitaxial Fi Lms Grown by Magnetron Sputtering. Thin Solid Films, 574, 99-102 (2015)

5. E. Greene, Review Article: Tracing the recorded history of thin-film sputter deposition: From the 1800s to 2017, J. Vac. Sci. Technol. A 35, 05 C204 (2017)

6. Flux Measurements F. Haase, D. Lundin, S. Bornholdt, and H. Kersten, On the Impact of Electron Temperature in Magnetron Sputtering Benchmarked with Energy, Contrib. Plasma Phys. 55, No. 10, $701-713$ (2015)

7. Yamamoto, Yuji, Peter Zaumseil, Tzanimir Arguirov, Martin Kittler, and Bernd Tillack. Low Threading Dislocation Density Ge Deposited on Si (100) Using RPCVD. Solid State Electronics ,60 (1), 2-6 (2011)

8. Samavati, Alireza, Z. Othaman, S. K. Ghoshal, and R. J. Amjad. Germanium Nanoislands Grown by Radio Frequency Magnetron Sputtering: Annealing Time Dependent Surface Morphology and Photoluminescence. Chinese Physics B, 22 (9): 98102(2013)

9. Shah, V. A., A. Dobbie, M. Myronov, and D. R. Leadley. Effect of Layer Thickness on Structural Quality of Ge Epilayers Grown Directly on Si (001). Thin Solid Films, 519 (22), 7911-17 (2011)

10. Ohring, Milton. 2002. "A Review of Material Science. In Materials Science of Thin Films: Deposition and Structure, edited by John L. Vossen, Second Edi, 794. San Diego, USA: Academic Press

11. Evcimen Duygulu, N., A. O. Kodolbas, and A. Ekerim. Effects of Argon Pressure and R.f. Power on Magnetron Sputtered Aluminum Doped ZnO Thin Films. Journal of Crystal Growth, 394,116-25 (2014)

12. Chaoumead, Accarat, Youl Moon Sung, and Dong Joo Kwak. 2012. The Effects of RF Sputtering Power and Gas Pressure on Structural and Electrical Properties of ITiO Thin Film, Advances in Condensed Matter Physics, 651587, 1- 7 (2012)

13. Gatzen, Hans H., Volker Saile, and Jürg Leuthold. Micro and Nano Fabrication: Tools and Processes. Micro and Nano Fabrication: Tools and Processes. New York: Springer (2015)

14. Belfedal, Abdelkader, Yahya Bouizem, Jamal Dine Sib, and Larbi Chahed. Films Thickness Effect on Structural and Optoelectronic Properties of Hydrogenated Amorphous Germanium (a-Ge:H). Journal 
of Non-Crystalline Solids, 358 (11), 1404-9 (2012)

15. Moreno, M., A. Torres, R. Ambrosio, P. Rosales, A. Heredia, A. Kosarev, E. Torres, C. Zuniga, C. Reyes Betanzo, and M. Dominguez. Deposition and Characterization of Polymorphous Germanium Films Prepared by Low Frequency PECVD. Journal of Non-Crystalline Solids 358 (17), 2099-2102 (2012)

16. Li, Lam Mui, Azmizam Manie, Shain Farah Lyana, Afishah Alias, and Saafie Salleh. Effect of Sputtering Pressure on the Structureal and Optical Properties of ZnO Films Deposited on Flexible Substrate. Jurnal Teknologi, 7 (75): 45-50 (2015)

17. Chinmulgund, M., R. B. Inturi, and J. A. Barnard. Effect of Ar Gas Pressure on Growth, Structure, and Mechanical Properties of Sputtered Ti, Al, TiAl, and Ti3Al Films. Thin Solid Films, 270 (1-2): 260-63 (1995)

\section{Figures}

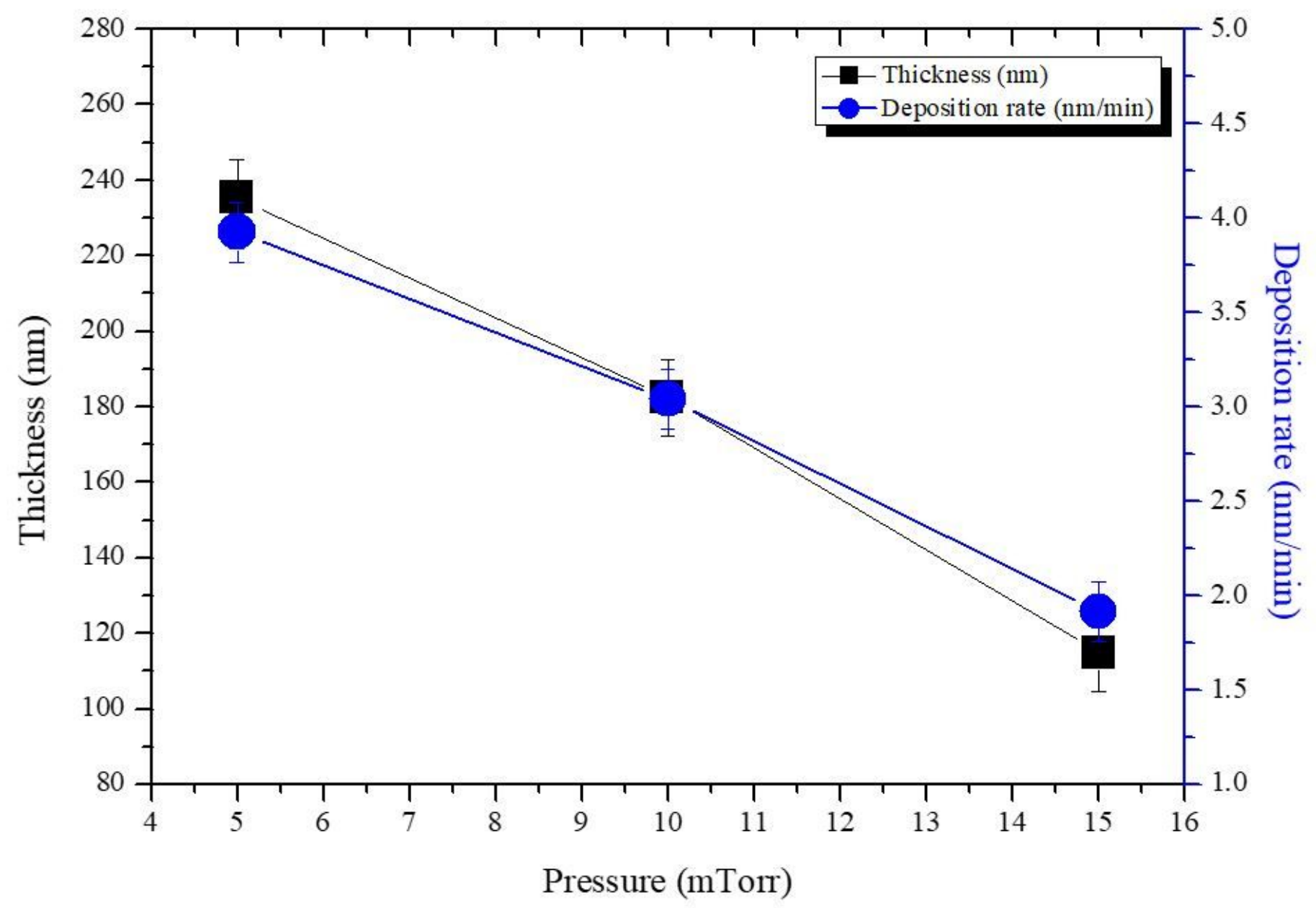

Figure 1

Thickness and deposition rate of Ge thin films at different gas pressures. 


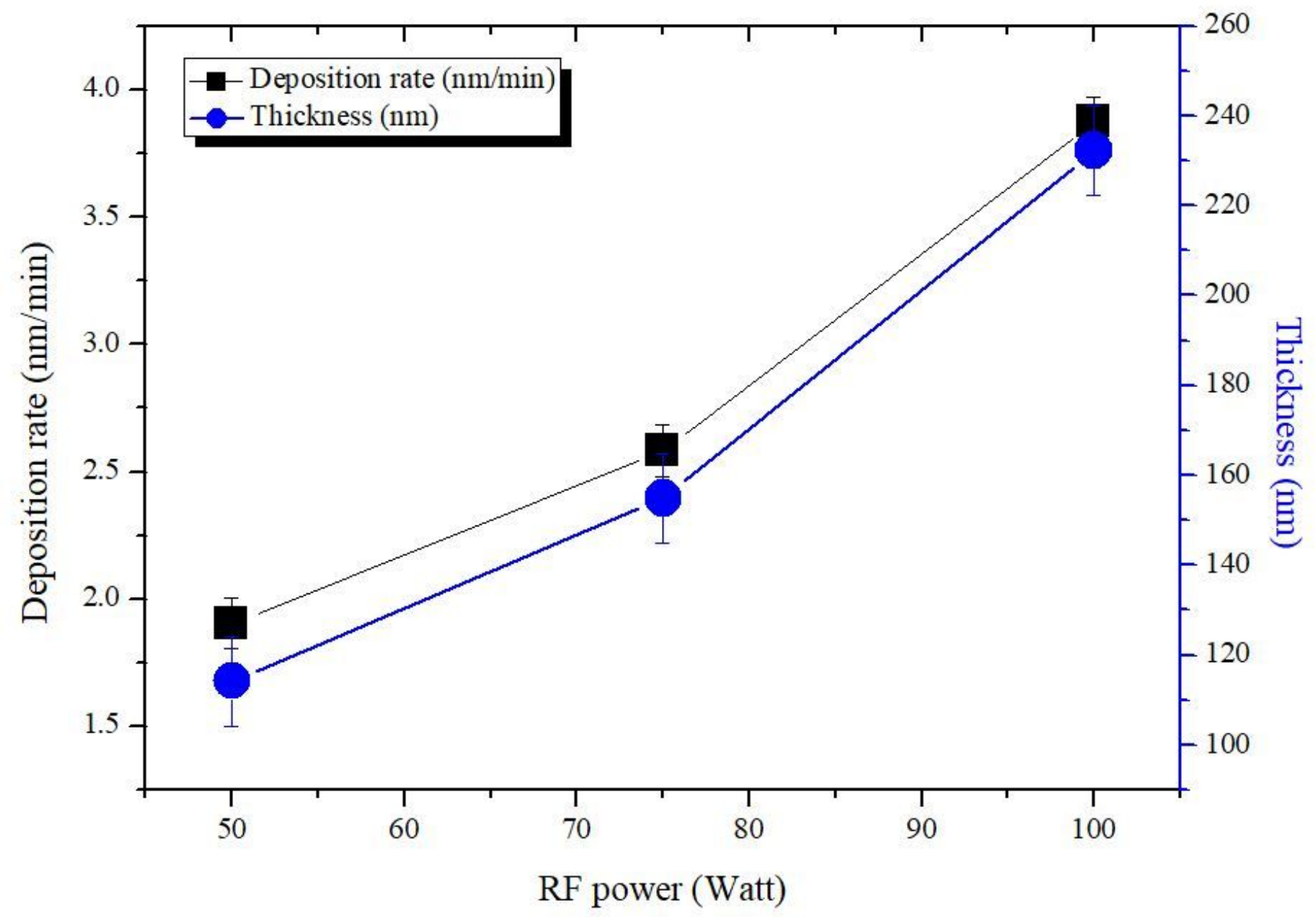

Figure 2

Thickness and deposition rate of Ge thin film at different RF powers. 
(a)

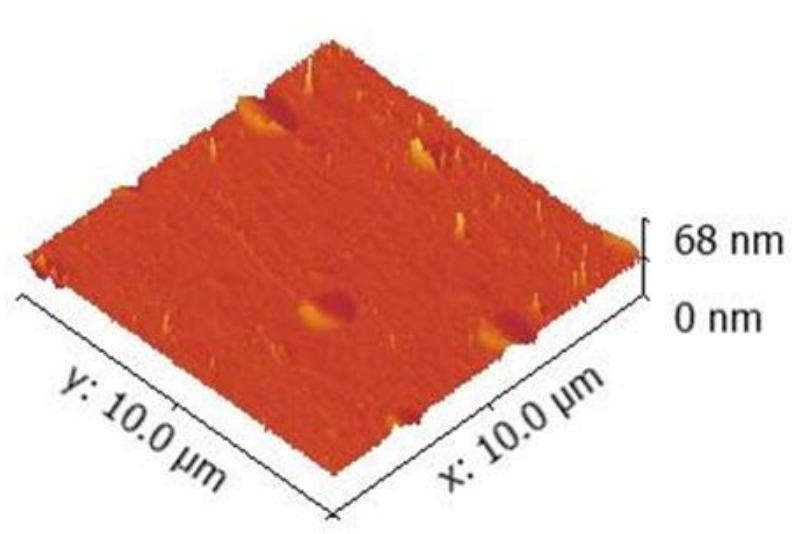

(b)

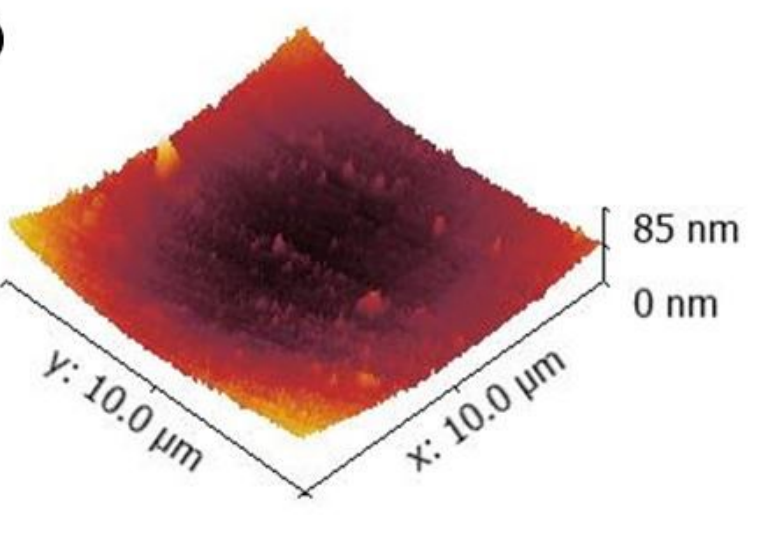

(c)

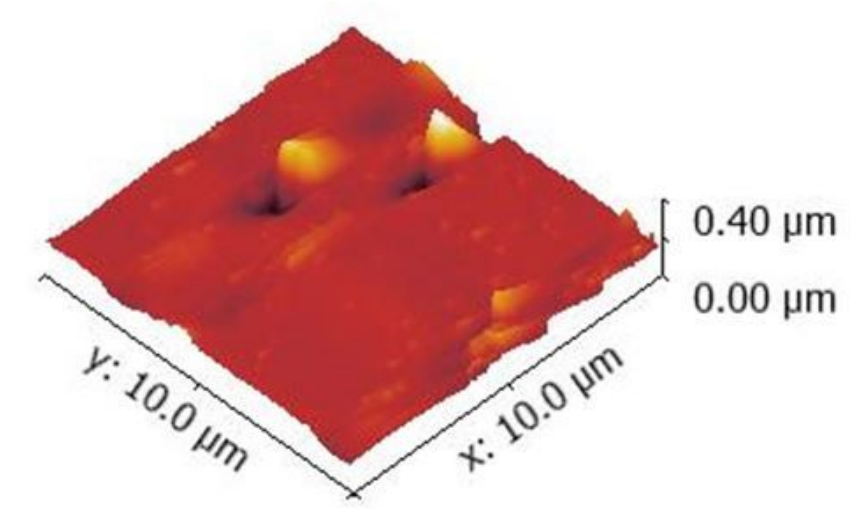

Figure 3

(a) AFM scan images at gas pressure of 5 mTorr, (b) 10 mTorr, and (c) 15 mTorr. 

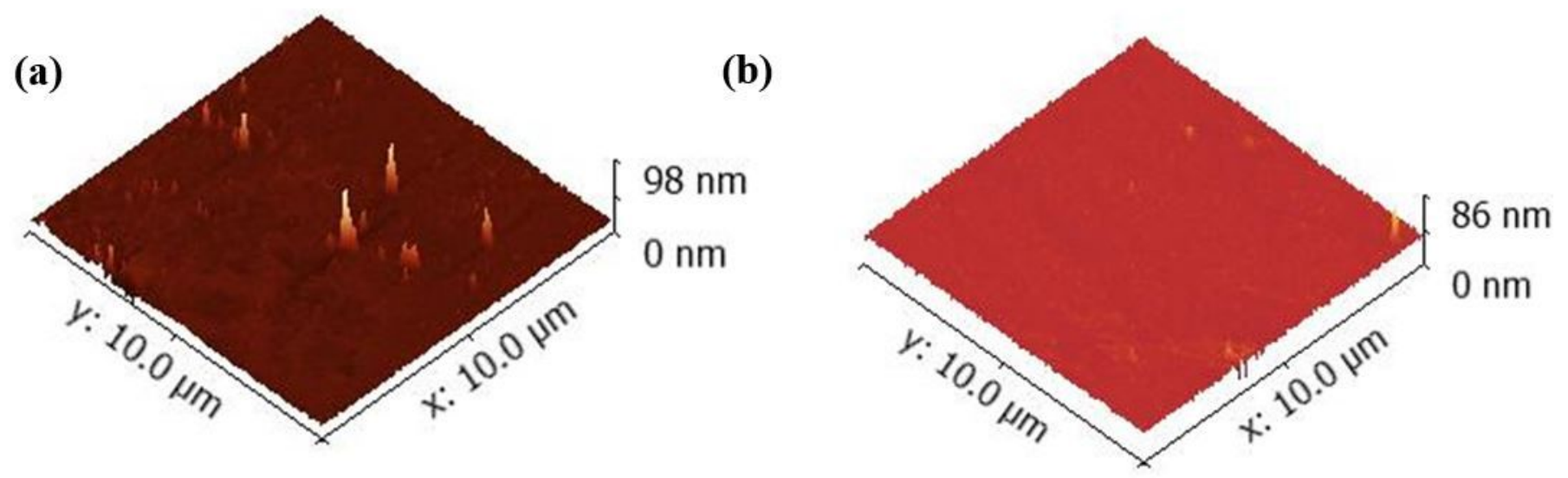

(c)

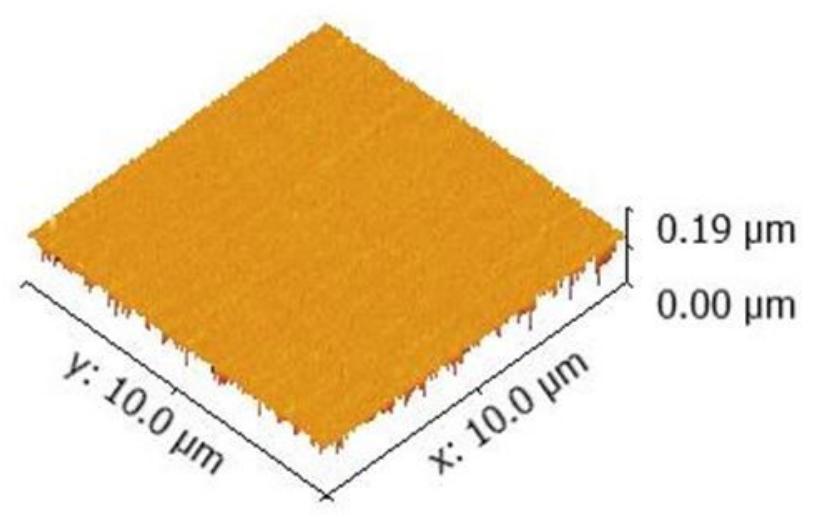

Figure 4

AFM scan images at RF power of (a) 50 Watt, (b) 75 Watt, and (c) 100 Watt. 
(a)

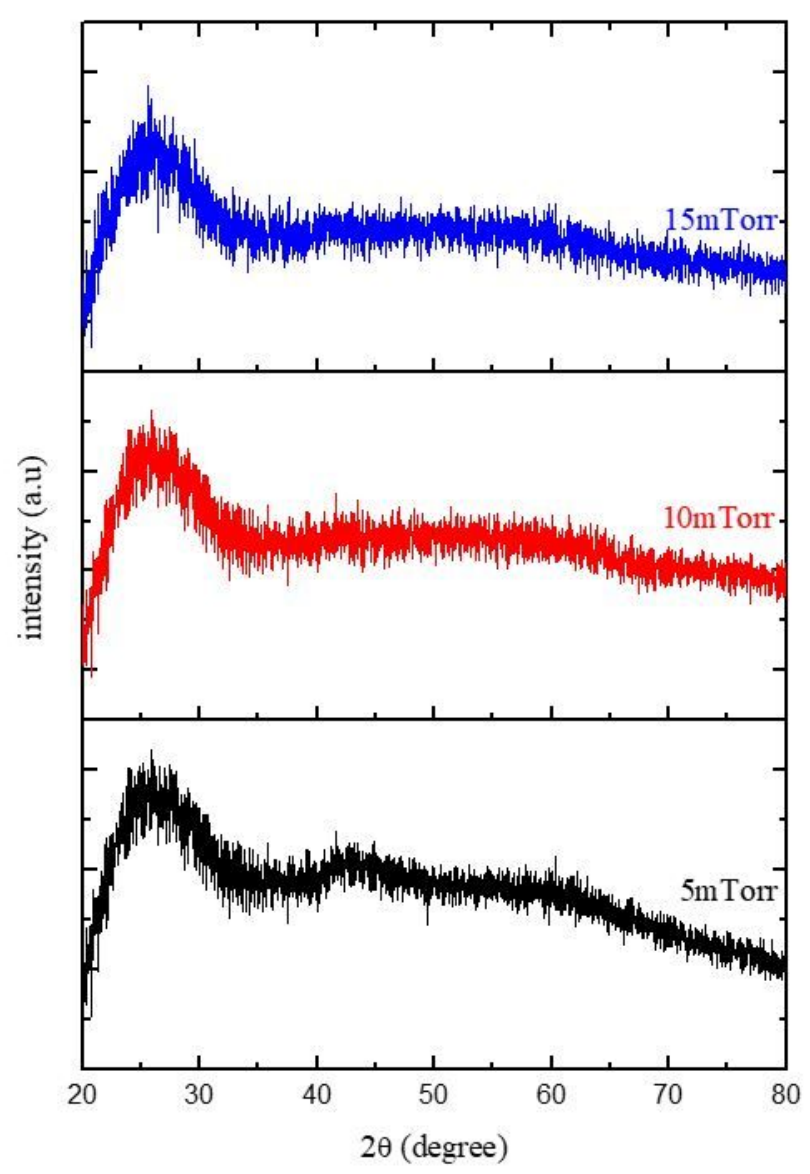

(b)

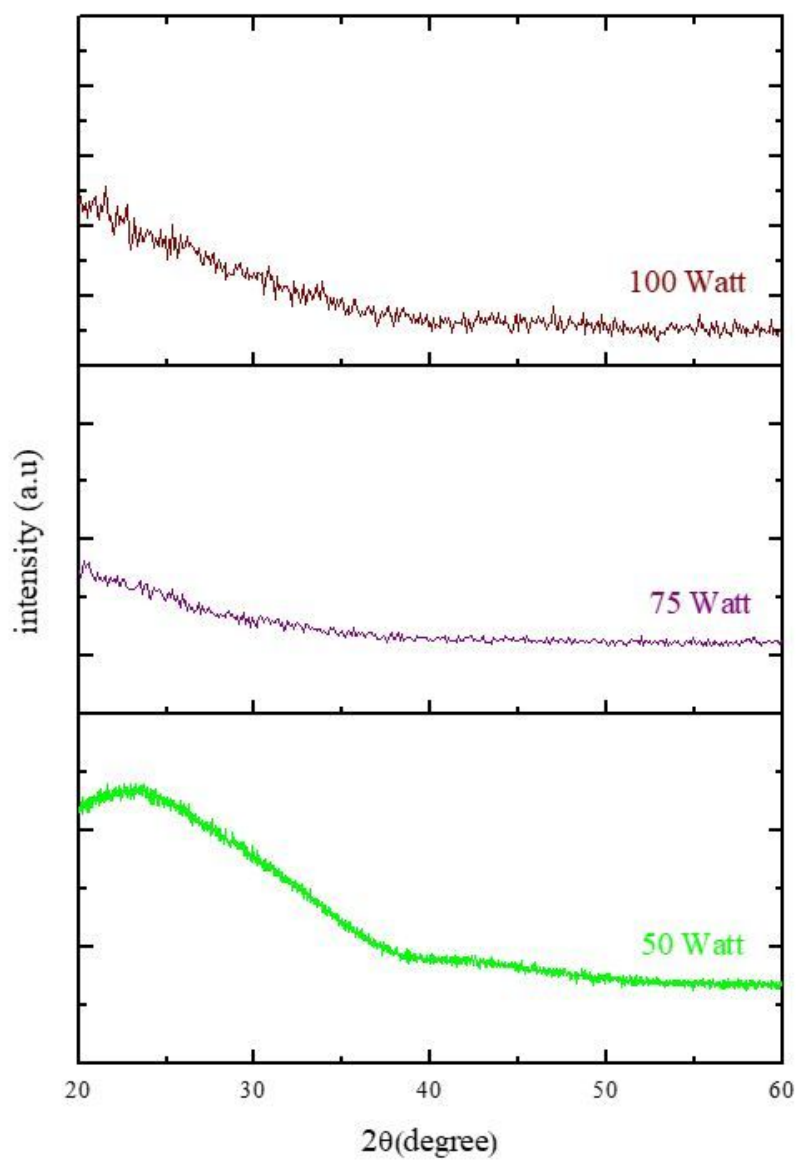

Figure 5

XRD analysis of Ge thin film for different (a) gas pressure and (b) RF power. 